\title{
Short-term Outcomes After Upfront Chemotherapy Followed by Curative Surgery in Metastatic Colon Cancer: A Comparison With Upfront Surgery Patients
}

\author{
Myung Hyun Han ${ }^{1}$, Youn Young Park ${ }^{2}$, Shiva Pratap ${ }^{3}$, Yoon Dae Han ${ }^{4}$, Min Soo Cho ${ }^{1}$, Hyuk Hur ${ }^{1}$, \\ Byung Soh Min ${ }^{1}$, Kang Young Lee ${ }^{1}$, Nam Kyu Kim ${ }^{1}$ \\ ${ }^{1}$ Department of Surgery, Yonsei University College of Medicine, Seoul; ${ }^{2}$ Department of Surgery, Uijeongbu St. Mary's Hospital, College of \\ Medicine, The Catholic University of Korea, Uijeongbu, Korea; ${ }^{3}$ Department of Surgery, Christian Medical College and Hospital, Vellore, \\ Tamil Nadu, India; ${ }^{4}$ Department of Surgery, Yonsei University College of Medicine, Yongin Severance Hospital, Yongin, Korea
}

Purpose: Upfront systemic chemotherapy with target agents has been recommended for patients with stage IV colon cancer. Some with partial response are considered for curative resection. There is high risk of developing postoperative complications following upfront systemic chemotherapy. We aimed to evaluate short-term perioperative outcomes of curative surgery after upfront chemotherapy in comparison with upfront surgery in patients with metastatic colon cancer.

Methods: Between January 2010 and October 2015, 146 patients (80 in the surgery first group, 66 in the upfront chemotherapy group) who underwent surgical resection before or after systemic chemotherapy for metastatic colon cancer were included in the present study. All decisions for treatment were made through a multidisciplinary team. Postoperative clinical outcomes and complications were analyzed to compare the groups.

Results: There was no difference between the 2 groups in terms of postoperative clinical outcomes. Overall complication rates were not different between the groups (surgery first group: $46.3 \%$ vs. upfront chemotherapy group: $60.6 \%$; $\mathrm{P}=$ 0.084). When classified according to the Clavien-Dindo method, there was no difference between the 2 groups in terms of major complications (grade 3 or more) (surgery first group: 18.9\% vs. upfront chemotherapy group: $27.5 \%$; $\mathrm{P}=0.374$ ).

Conclusion: There was no significant increase in major postoperative complications in metastatic colon cancer patients who received upfront chemotherapy followed by curative surgery. Careful patient selection and treatment planning are important.

Keywords: Colonic neoplasms; Neoplasm metastasis; Antineoplastic agents; Postoperative complications

\section{INTRODUCTION}

At the time of diagnosis, approximately $20 \%-25 \%$ of colorectal cancer patients already have metastasis. In these individuals, the

Received: November 30, 2018 - Accepted: March 4, 2019

Correspondence to: Nam Kyu Kim, M.D.

Division of Colorectal Surgery, Department of Surgery, Yonsei University College of Medicine, 50-1 Yonsei-ro, Seodaemun-gu, Seoul 03722, Korea Tel: +82-2-2228-2117, Fax: +82-2-313-8289, E-mail: namkyuk@yuhs.ac ORCID code: https://orcid.org/0000-0003-0639-5632

(C) 2019 The Korean Society of Coloproctology

This is an open-access article distributed under the terms of the Creative Commons Attribution NonCommercial License (http://creativecommons.org/licenses/by-nc/4.0) which permits unrestricted noncommercial use, distribution, and reproduction in any medium, provided the original work is properly cited. treatment strategy will be palliative or curative according to disease status [1]. In recent years, even unresectable metastatic lesions can be resected if the tumor response is favorable to previous systemic chemotherapy. Adam et al. [2] found that, among 1,104 initially unresectable colorectal liver metastases patients, 138 with good response (12.5\%) underwent hepatic resection after an average of 10 sessions of chemotherapy, and a 5-year survival rate of $33 \%$ was achieved versus a rate of $5 \%$ in patients who did not undergo resection. In some cases of resectable metastasis, upfront systemic chemotherapy is recommended for various reasons. First, upfront chemotherapy can play a role as an in vivo chemosensitivity assay and can be used in conjunction with metabolic imaging (e.g., fluorodeoxyglucose - positron emission tomography) to reflect differences in the biologic tumor associated 
with various responses. Further, chemically reducing the tumor prior to surgery can lessen the frequency of tumor cell shedding during surgery and improve the rate of R0 resection [3]. The goal of systemic chemotherapy for these patients is to cure the disease or prolong survival and maintain quality of life as long as possible [1].

Several developments have improved systemic chemotherapy in patients with metastatic colorectal cancer. Introduction of combination chemotherapy protocols and addition of new biological target agents such as bevacizumab and cetuximab have led to significant advances in tumor response after chemotherapy for metastatic colorectal cancer patients [4]. In particular, the target agent acts on a specific molecule of cancer cells to exert its anticancer effect, which is less harmful to normal cells than other types of chemotherapy and has the potential to cause fewer side effects. In a recent review, addition of a biologic agent to conventional chemotherapy increased the rate of conversion to a resectable state in patients with unresectable metastatic colorectal cancer [5].

However, systemic chemotherapy alone rarely cures metastatic colorectal cancer. In patients with metastatic colorectal cancer, curative resection (R0) is the only therapeutic option for longterm survival [6]. In addition, in potentially resectable stage IV colon cancer patients with good tumor response after upfront chemotherapy, R0 resection can be achieved [7].

However, administration of chemotherapy may lead to higher perioperative morbidity. Oxaliplatin is related to sinusoidal liver lesions, and irinotecan is related to steatohepatitis. We also do not yet fully understand the potential toxicity of antiangiogenesis agents like bevacizumab [8]. In addition, combined resection for primary tumor and metastasis usually requires many surgical procedures and long operation times. Furthermore, patients may receive a long duration of upfront chemotherapy or chemoradiation therapy. These concerns may place a burden on the surgeon in making decisions about the operation regarding timing of surgery after upfront chemotherapy. Additionally, the optimal timing of surgery and cessation of systemic chemotherapy prior to surgery are not well-established.

The aim of our study was to evaluate perioperative clinical short-term outcomes of curative surgery after upfront chemotherapy for patients with metastatic colon cancer.

\section{METHODS}

\section{Patients}

We retrospectively analyzed 146 patients diagnosed with metastatic colon cancer who underwent surgical resection before or after systemic chemotherapy at Yonsei University Severance Hospital, South Korea, between January 2010 and October 2015. Patients with peritoneal metastasis were excluded from the present study. We collected information on all study participants from their medical records and divided them into the 2 groups: the surgery first group of 80 initially 'resectable' patients who underwent surgical resection followed by adjuvant chemotherapy, and the upfront chemotherapy group of 66 initially 'potentially resectable' or 'unresectable' patients who underwent upfront chemotherapy followed by surgical resection. We retrospectively compared these 2 groups. To evaluate the distribution, number, and size of the lesions before initiation of treatment, image examinations of the thoracic, abdominal, and pelvic areas were performed. All decisions for treatment were made by a multidisciplinary team composed of surgeons, medical oncologists, radiation oncologists, and radiologists. To achieve improved accuracy in decision-making and in treatment plans as well as better surgical and oncologic outcomes and increased patient satisfaction, all patients were evaluated with consideration of the various aspects during a multidisciplinary team conference. Resectability of the primary tumor and metastatic lesions was mainly assessed via reinterpretation by and discussion of the imaging results by radiologists and surgeons. We initially identified the primary tumor as 'unresectable' when it showed extensive involvement of a major structure like the vena cava, superior mesenteric vessel, pancreas, or duodenum. For hepatic metastatic lesions, when the remnant parenchyma of the liver was expected to be less than $30 \%$ of the total volume after resection, we defined this as 'unresectable.' When resection was needed rather than lobectomy, or if a patient had insufficient respiratory function, we categorized the cancer as 'unresectable' lung metastasis [9]. For patients with 'unresectable' or 'potentially resectable' tumors, a chemotherapeutic agent was administered according to the guidelines of National Health Insurance of Korea. The physician chose the target agent in consultation with the patient according to the results of a K-ras mutation test. Cetuximab or bevacizumab was used for patients without the K-ras mutation, and bevacizumab was chosen for patients with the Kras mutation. During patient follow-up, imaging studies were repeated to investigate treatment response and resectability. According to the RECIST (response evaluation criteria in solid tumor) [10], we defined a 'partial response' as a $\geq 50 \%$ decrease in tumor size on imaging studies. 'Progressive disease' was defined as a $>25 \%$ increase in tumor size or detection of newly developed lesions. Otherwise, cases were defined as 'stable disease.' In this study, 33 patients (50\%) showed 'partial response', 32 patients (48.5\%) had 'stable disease', and only 1 patient (1.5\%) showed 'progressive disease' based on one newly developed liver metastasis on imaging during follow-up. When a tumor converted to resectable during follow-up, we considered surgery with a curative intent. This study was approved by the Institutional Review Board (IRB) of Severance Hospital Yonsei University Health System (IRB No. 2018-0566-001). The need for informed consent was waived by the IRB.

\section{Clinical variables}

Data on patient and tumor characteristics, upfront chemotherapy regimens, operative factors, and clinical factors related to postoperative outcome were collected from medical records. Patient and 
tumor characteristics comprised age; sex; weight; height; body mass index (BMI); American Society of Anesthesiologists (ASA) physical status classification; primary colon cancer location; organ with metastasis; tumor, lymph node $(\mathrm{N})$, and metastasis (M) stages in accordance with the American Joint Committee on Cancer staging [11]; and histology of the tumor. Upfront chemotherapy regimens were evaluated for agents used, duration and cycles of therapy, and time from completion of chemotherapy to surgery. Operative variables were surgical approach method, surgical procedures for primary colon cancer, surgical procedures for metastatic lesions, and open conversion.

\section{Operative management}

All patients underwent primary resection of colon cancer according to location, and resection of metastatic lesions was performed. Some patients underwent a staged operation following consideration of the location and size of the metastatic lesions and the comorbidity and condition of the patient. In patients with more than 2 organ metastases, simultaneous resection was performed when possible. However, in most cases, a staged operation was performed considering the width of resection. Meanwhile, if it was impossible to perform resection or if the risk was greater than the anticipated benefit, a second operation was not performed.

\section{Postoperative outcomes}

Postoperative short-term clinical outcomes of time to first fluid intake, time to first flatus, and length of hospital stay were evaluated. Postoperative complications were evaluated and graded using the Clavien-Dindo classification system [12], a previously defined grading system for surgical complications.

\section{Statistical analysis}

We performed all statistical analyses using IBM SPSS Statistics ver. 23.0 (IBM Co., Armonk, NY, USA). Categorical variables were analyzed using the chi-square or Fisher exact test, while continuous variables were analyzed using Student t-test or MannWhitney U-test. A significance level (P-value) less than 0.05 was considered statistically significant.

\section{RESULTS}

\section{Patient and tumor characteristics}

Patient and tumor characteristics are listed in Table 1. There were more T4 patients in the Surgery first group (surgery first group: $33.8 \%$ vs. upfront chemotherapy group: $6.1 \%$ ) and more T3 patients in the upfront chemotherapy group (surgery first group: $61.3 \%$ vs. upfront chemotherapy group: $83.3 \%$ ), possibly due to tumor shrinkage caused by upfront chemotherapy. In comparison, age, sex, weight, height, BMI, ASA physical status classification, primary tumor site, metastasis site, $\mathrm{N}$ stage, $\mathrm{M}$ stage, and histology did not differ significantly between the 2 groups. In both groups, the most frequent primary colon cancer site was the
Table 1. Baseline demographic and clinicopathological characteristics

\begin{tabular}{|c|c|c|c|}
\hline Characteristic & $\begin{array}{l}\text { Surgery first } \\
\quad(n=80)\end{array}$ & $\begin{array}{c}\text { Upfront } \\
\text { chemotherapy } \\
(\mathrm{n}=66)\end{array}$ & P-value \\
\hline Age (yr) & $61.31 \pm 11.91$ & $59.00 \pm 9.20$ & 0.199 \\
\hline Sex & & & 0.937 \\
\hline Male & $49(61.3)$ & $40(60.6)$ & \\
\hline Female & $31(38.8)$ & $26(39.4)$ & \\
\hline Weight (kg) & $62.37 \pm 9.88$ & $61.56 \pm 11.26$ & 0.647 \\
\hline Height (cm) & $162.87 \pm 8.08$ & $161.95 \pm 7.09$ & 0.471 \\
\hline Body mass index $\left(\mathrm{kg} / \mathrm{m}^{2}\right)$ & $23.41 \pm 2.66$ & $23.34 \pm 3.17$ & 0.879 \\
\hline ASA PS classification $\geq$ III & $14(17.5)$ & $16(24.2)$ & 0.316 \\
\hline K-ras status & & & 0.031 \\
\hline Wild type & $22(27.5)$ & $32(48.5)$ & \\
\hline Mutant & $28(35.0)$ & $15(22.7)$ & \\
\hline Unknown & $30(37.5)$ & $19(28.8)$ & \\
\hline Primary tumor site & & & 0.605 \\
\hline Cecum & $3(3.8)$ & $2(3.0)$ & \\
\hline Ascending colon & $19(23.8)$ & $12(18.2)$ & \\
\hline Hepatic flexure & $5(6.3)$ & $1(1.5)$ & \\
\hline Transverse colon & $4(5.0)$ & $4(6.1)$ & \\
\hline Splenic flexure & $1(1.3)$ & $0(0)$ & \\
\hline Descending colon & $7(8.8)$ & $10(15.2)$ & \\
\hline Sigmoid colon & $40(50.0)$ & $37(56.1)$ & \\
\hline Ascending colon + sigmoid colon & $1(1.3)$ & $0(0)$ & \\
\hline \multicolumn{4}{|l|}{ Metastatic site } \\
\hline Liver & 74 (92.5) & 65 (98.5) & 0.128 \\
\hline Lung & $10(12.5)$ & $11(16.7)$ & 0.475 \\
\hline Para-aortic lymph node & $5(6.3)$ & $8(12.1)$ & 0.215 \\
\hline Adrenal gland & $0(0)$ & $1(1.5)$ & 1 \\
\hline Diaphragm & $1(1.3)$ & $0(0)$ & 1 \\
\hline T stage & & & $<0.001$ \\
\hline T0, no residual cancer & $1(1.3)$ & $4(6.1)$ & \\
\hline $\mathrm{T} 1$ & $0(0)$ & $0(0)$ & \\
\hline $\mathrm{T} 2$ & $3(3.8)$ & $3(4.5)$ & \\
\hline T3 & 49 (61.3) & 55 (83.3) & \\
\hline T4a & $21(26.3)$ & $4(6.1)$ & \\
\hline T4b & $6(7.5)$ & $0(0)$ & \\
\hline N stage & & & 0.43 \\
\hline NO & $19(23.8)$ & $16(24.2)$ & \\
\hline $\mathrm{N} 1 \mathrm{a}$ & $15(18.8)$ & $10(15.2)$ & \\
\hline N1b & $20(25.0)$ & $20(30.3)$ & \\
\hline N1C & $3(2.2)$ & $1(1.5)$ & \\
\hline $\mathrm{N} 2 \mathrm{a}$ & $9(11.3)$ & $13(19.7)$ & \\
\hline $\mathrm{N} 2 \mathrm{~b}$ & $14(17.5)$ & $6(9.1)$ & \\
\hline
\end{tabular}

(Continued to the next page) 
Table 1. Continued

\begin{tabular}{lccc}
\hline Characteristic & $\begin{array}{c}\text { Surgery first } \\
(\mathrm{n}=80)\end{array}$ & $\begin{array}{c}\text { Upfront } \\
\text { chemotherapy } \\
(\mathrm{n}=66)\end{array}$ & P-value \\
\hline M stage & $66(82.5)$ & $47(71.2)$ & 0.105 \\
M1a & $14(17.5)$ & $19(28.8)$ & \\
M1b & & & 0.372 \\
Histology & $3(3.8)$ & $4(6.1)$ & \\
Well-differentiated & $74(92.5)$ & $59(89.4)$ & \\
Moderately differentiated & $1(1.3)$ & $3(4.5)$ & \\
Poorly differentiated & $2(2.5)$ & $0(0)$ & \\
Mucinous &
\end{tabular}

Values are presented as mean \pm standard deviation or number (\%). ASA PS, American Society of Anesthesiologists physical status.

Table 2. Details of chemotherapy $(n=66)$

\begin{tabular}{|c|c|}
\hline Variable & Value \\
\hline \multicolumn{2}{|l|}{ Regimen of upfront chemotherapy } \\
\hline FOLFOX & $38(57.6)$ \\
\hline FOLFIRI $^{\mathrm{b}}$ & $27(40.9)$ \\
\hline XELOXC & $1(1.5)$ \\
\hline Use of molecular target agent & $44(66.7)$ \\
\hline Bevacizumab & $23(52.3)$ \\
\hline Cetuximab & $20(45.5)$ \\
\hline Aflibercept & $1(2.3)$ \\
\hline Duration of chemotherapy (mo) & $3(0-32)$ \\
\hline Time interval between chemotherapy and surgery (wk) & $6.4(3.7-57)$ \\
\hline Cycles of chemotherapy & $6(2-46)$ \\
\hline
\end{tabular}

Values are presented as number (\%) or median (range).

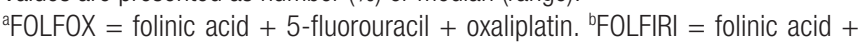
5-fluorouracil + irinotecan. ${ }^{~}$ XELOX $=$ capecitabine + oxaliplatin.

sigmoid colon (surgery first group: $50.0 \%$ vs. upfront chemotherapy group: $56.1 \%$ ), and the most frequent metastatic organs were the liver and lungs (surgery first group: $92.5 \%$ and $12.5 \%$, respectively, vs. upfront chemotherapy group: $98.5 \%$ and $16.7 \%$ ).

Upfront chemotherapy was administered to all upfront chemotherapy group patients (Tables 2, 3). Combination therapies containing oxaliplatin or irinotecan (folinic acid +5 -fluorouracil + oxaliplatin [FOLFOX]; capecitabine + oxaliplatin [XELOX]; or folinic acid + 5-fluorouracil + irinotecan [FOLFIRI]) were adapted. Forty-four patients received the following target agents: bevacizumab $(\mathrm{n}=23 ; 52.3 \%)$, cetuximab $(\mathrm{n}=20 ; 45.5 \%)$, and aflibercept $(\mathrm{n}=1 ; 2.3 \%)$. The median duration of upfront chemotherapy was 3 months (range, 0-32 months), and the median cycle number for chemotherapy was 6 (range, $2-46$ cycles). Additionally, the median time interval from completion of upfront chemotherapy and combined surgery was 6.4 weeks (range, 3.7-
Table 3. Details of adjuvant chemotherapy

\begin{tabular}{|c|c|c|c|}
\hline Variable & $\begin{array}{l}\text { Surgery first } \\
\qquad(\mathrm{n}=80)\end{array}$ & $\begin{array}{c}\text { Upfront } \\
\text { chemotherapy } \\
(n=66)\end{array}$ & P-value \\
\hline Regimen of adjuvant chemotherapy & & & $<0.001$ \\
\hline FOLFOX & 65 (81.3) & $32(48.5)$ & \\
\hline FOLFIRI $\left.\right|^{b}$ & $3(3.8)$ & $29(43.9)$ & \\
\hline$X^{\prime} E^{\prime} O X^{c}$ & $3(3.8)$ & $1(1.5)$ & \\
\hline $\mathrm{FL}^{\mathrm{d}}$ & $1(1.3)$ & $0(0)$ & \\
\hline No adjuvant chemotherapy & $8(10.0)$ & $4(6.1)$ & \\
\hline Use of molecular target agent & & & $<0.001$ \\
\hline Cetuximab & $1(1.3)$ & $15(23.1)$ & \\
\hline Bevacizumab & $9(11.4)$ & $17(26.2)$ & \\
\hline No target agent & $70(87.5)$ & 34 (51.5) & \\
\hline Cycles of chemotherapy & $12(0-12)$ & $6(0-16)$ & $<0.001$ \\
\hline
\end{tabular}

Values are presented as number (\%) or median (range).

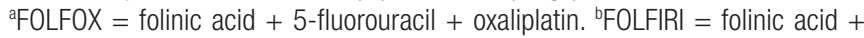
5 -fluorouracil + irinotecan. ${ }^{\text {CXELOX }}=$ capecitabine + oxaliplatin. ${ }^{\mathrm{d}} \mathrm{FL}=5$-fluorouracil + leucovorin.

57 weeks). In patients who received bevacizumab, the median chemotherapy duration was 2 months (range, 1-32 months), the median number of chemotherapy cycles was 6 (range, 4-46 cycles), and the median time interval for completion of chemotherapy and surgery was 6.8 weeks (range, 4-35 weeks). Because of the high risk of recurrence, systemic chemotherapy was performed in both groups after surgery.

\section{Operative management}

All patients underwent combined surgery for primary tumor and metastatic lesions (Table 4). Open surgery was most common in both groups (surgery first group: $60 \%$ vs. upfront chemotherapy group: $87.9 \%$ ). In the surgery first group, more laparoscopic surgery was performed (surgery first group: $40 \%$ vs. upfront chemotherapy group: 9.1\%). Conversion rates between the 2 groups were not significantly different (surgery first group: $0 \%$ vs. upfront chemotherapy group: $3 \%$ ). Hepatic resections were classified according to the segmental anatomy of the liver as defined by Couinaud [13]. We defined major liver resection as removal of 4 or more liver segments, and this type was performed more often in the upfront chemotherapy group (surgery first group: $8.1 \%$ vs. upfront chemotherapy group: $21.5 \%$ ). In surgery for lung metastasis, an initial combined operation was more common in the surgery first group (surgery first group: $70 \%$ vs. upfront chemotherapy group: 9.1\%). Meanwhile, a staged operation or no resection was performed in some cases based on the distribution of lesions or comorbidity of the patient. For liver metastasis, 5 staged liver resections were performed (surgery first group: $1.4 \%$ vs. upfront chemotherapy group: 6.2\%). For lung metastasis, 7 staged resections were conducted only in the upfront chemotherapy 
Volume 35, Number 6, 2019

Ann Coloproctol 2019;35(6):327-334

Table 4. Operative management

\begin{tabular}{|c|c|c|c|}
\hline Variable & $\begin{array}{l}\text { Surgery first } \\
\quad(n=80)\end{array}$ & $\begin{array}{l}\text { Upfront } \\
\text { chemotherapy } \\
(n=66)\end{array}$ & P-value \\
\hline Open conversion & $0(0)$ & $2(3.0)$ & 0.203 \\
\hline Surgical approach & & & $<0.001$ \\
\hline Open & $48(60.0)$ & $58(87.9)$ & \\
\hline Laparoscopic & $32(40.0)$ & $6(9.1)$ & \\
\hline Robotic & $0(0)$ & $2(3.0)$ & \\
\hline Simultaneous resection & $76(95.0)$ & $52(78.8)$ & 0.003 \\
\hline Staged resection for metastasis & $1(1.3)$ & $11(16.7)$ & 0.001 \\
\hline $\begin{array}{l}\text { Failed staged resection for } \\
\text { metastasis }\end{array}$ & $3(3.8)$ & $3(4.5)$ & 1.000 \\
\hline Surgery for primary tumor & & & 0.218 \\
\hline Anterior resection & $40(50.0)$ & $37(56.1)$ & \\
\hline Right hemicolectomy & $29(36.3)$ & $16(24.2)$ & \\
\hline Left hemicolectomy & $8(10.0)$ & $12(18.2)$ & \\
\hline $\begin{array}{l}\text { Segmental resection of } \\
\text { transverse colon }\end{array}$ & $1(1.3)$ & $1(1.5)$ & \\
\hline $\begin{array}{l}\text { Right hemicolectomy + anterior } \\
\text { resection }\end{array}$ & $2(2.5)$ & $0(0)$ & \\
\hline Surgery for liver metastasis & & & $<0.001$ \\
\hline $\begin{array}{l}\text { Major liver resection } \\
\text { ( } \geq 4 \text { segments) }\end{array}$ & $6(8.1)$ & $14(21.5)$ & \\
\hline $\begin{array}{l}\text { Minor liver resection } \\
\text { (<4 segments) }\end{array}$ & $25(33.7)$ & $31(47.7)$ & \\
\hline Wedge resection or RFA only & $42(56.7)$ & $16(24.6)$ & \\
\hline Staged resection & $1(1.4)$ & $4(6.2)$ & \\
\hline Surgery for lung metastasis & & & 0.008 \\
\hline Wedge resection under VATS & $7 / 10(70.0)$ & $1 / 11(9.1)$ & \\
\hline No resection & $3 / 10(30.0)$ & 3/11 (27.2) & \\
\hline Staged resection & 0/10 (0) & 7/11 (63.6) & \\
\hline
\end{tabular}

Values are presented as number (\%).

RFA, radiofrequency ablation; VATS, video-assisted thoracic surgery.

group (surgery first group: $0 \%$ vs. upfront chemotherapy group: 63.6\%). However, in 6 additional cases, planned staged resection for lung metastasis could not be performed due to poor patient condition and progression of metastasis (surgery first group: $30 \%$ vs. upfront chemotherapy group: $27.2 \%$ ). All patients who experienced metastasis to para-aortic lymph nodes underwent curative para-aortic lymph node dissection.

\section{Postoperative outcomes}

There was no difference between the 2 groups in postoperative clinical course (Table 5). The median length of postoperative care was 10 days (range, 5-77 days) in the surgery first group and 12
Table 5. Postoperative outcomes

\begin{tabular}{|c|c|c|c|}
\hline Variable & $\begin{array}{l}\text { Surgery first } \\
\quad(n=80)\end{array}$ & $\begin{array}{l}\text { Upfront } \\
\text { chemotherapy } \\
(n=66)\end{array}$ & P-value \\
\hline Time to first fluid intake (day) & $2(1-25)$ & $2(1-6)$ & 0.570 \\
\hline Time to first flatus (day) & $4(1-9)$ & $4(1-7)$ & 0.770 \\
\hline Length of hospital stay (day) & $10(5-77)$ & $12(4-47)$ & 0.470 \\
\hline Reoperation & $1(1.3)$ & $1(1.5)$ & 1.000 \\
\hline Complications & $37(46.3)$ & $40(60.6)$ & 0.084 \\
\hline Fever & $15(18.8)$ & $20(30.3)$ & 0.104 \\
\hline Ascites & $2(2.5)$ & $10(15.2)$ & 0.006 \\
\hline Chyle leakage & $3(3.8)$ & $4(6.1)$ & 0.701 \\
\hline lleus & $14(17.5)$ & $8(12.1)$ & 0.366 \\
\hline Cardiac & $0(0)$ & $2(3)$ & 0.203 \\
\hline Pulmonary & $8(10.0)$ & $16(24.2)$ & 0.021 \\
\hline Urinary tract infection & $0(0)$ & $1(1.5)$ & 0.452 \\
\hline Voiding difficulty & $3(3.8)$ & $2(3.0)$ & 1.000 \\
\hline Renal & $1(1.3)$ & $1(1.5)$ & 1.000 \\
\hline Delirium & $1(1.3)$ & $1(1.5)$ & 1.000 \\
\hline Bleeding & $0(0)$ & $1(1.5)$ & 0.452 \\
\hline Anastomotic leakage & $1(1.3)$ & $2(3.0)$ & 0.590 \\
\hline Bile leakage & $0(0)$ & $2(3.0)$ & 0.203 \\
\hline Superficial SSI & $6(7.5)$ & $7(10.6)$ & 0.512 \\
\hline Organ/space SSI & $2(2.5)$ & $4(6.1)$ & 0.410 \\
\hline $\begin{array}{l}\text { Life-threatening complication } \\
\text { requiring ICU management (G } \mathrm{IV} \text { ) }\end{array}$ & $2(2.5)$ & $1(1.5)$ & 1.000 \\
\hline $\begin{array}{l}\text { Reoperation due to anastomotic } \\
\text { leakage }\end{array}$ & $1(1.3)$ & $0(0)$ & \\
\hline Reoperation due to bile leakage & $0(0)$ & $1(1.5)$ & \\
\hline Septic pneumonia & $1(1.3)$ & $0(0)$ & \\
\hline Clavien-Dindo classification & & & 0.263 \\
\hline I & $3(8.1)$ & $6(15.0)$ & \\
\hline$\|$ & 27 (72.9) & $23(57.5)$ & \\
\hline$\| \mathrm{A}$ & $5(13.5)$ & $10(25.0)$ & \\
\hline$\| I I B$ & $0(0)$ & $0(0)$ & \\
\hline IVA & $1(2.7)$ & $1(2.5)$ & \\
\hline IVB & $0(0)$ & $0(0)$ & \\
\hline V & $1(2.7)$ & $0(0)$ & \\
\hline Total & 37 & 40 & \\
\hline Major complication ( $\mathrm{G} \geq \| \mathrm{I} A)$ & $7(18.9)$ & $11(27.5)$ & 0.374 \\
\hline
\end{tabular}

Values are presented as median (range) or number (\%).

SSI, Surgical site infection; ICU, intensive care unit.

days (range, 4-47 days) in the upfront chemotherapy group. A total of 58 postoperative complications developed in 37 patients 
$(46.3 \%)$ in the surgery first group versus 82 postoperative complications in 40 patients $(60.6 \%)$ in the upfront chemotherapy group (Table 5). However, there was no significant difference in overall complication rate between the 2 groups (surgery first group: $46.3 \%$ vs. upfront chemotherapy group: $60.6 \%$; $\mathrm{P}=0.084$ ). There was a notable difference in incidence of postoperative ascites (surgery first group: $2.5 \%$ vs. upfront chemotherapy group: $15.2 \%$; $=0.006$ ) and pulmonary complications (surgery first group: $10 \%$ vs. upfront chemotherapy group: $24.2 \%$; $\mathrm{P}=0.021$ ), as both occurred more often in the upfront chemotherapy group. Conversely, however, there was no difference between the 2 groups in terms of surgical site infection, bowel anastomotic leakage or bile leakage, or postoperative bleeding after surgery. In the upfront chemotherapy group, there was no bleeding or anastomotic leakage in any patient who received bevacizumab. There was no difference between the 2 groups when classified according to the Clavien-Dindo method ( $\mathrm{P}=0.263$ ). There was no difference between the 2 groups in terms of major complications of grade III or higher (surgery first group: $18.9 \%$ vs. upfront chemotherapy group: $27.5 \%$; $\mathrm{P}=0.374$ ). There was one case that needed intensive care unit (ICU) admission following reoperation in both groups (surgery first group: $1.3 \%$ vs. upfront chemotherapy group: $1.5 \%)$. Reoperation was performed due to bowel anastomotic leakage in the surgery first group and due to bile leakage in the upfront chemotherapy group. One patient in the surgery first group died within 30 days of surgery after ICU care due to postoperative renal failure and pulmonary sepsis.

\section{DISCUSSION}

Although metastatic colorectal cancer is generally incurable, resection of oligometastatic disease has curative potential [14]. Furthermore, with the development of systemic chemotherapy, patients with 'unresectable' or 'potentially resectable' metastatic colorectal cancer are more likely to undergo surgical resection following upfront chemotherapy. This conversion therapy has been the subject of recent attention.

It may be natural for surgical oncologists to actively consider surgical resection for patients with 'unresectable' or 'potentially resectable' metastatic colorectal cancer. However, with extensive surgical resection and toxicity of systemic chemotherapy, it may be difficult to make aggressive decisions due to concerns about patient recovery after surgery. In addition, the optimal timing of appropriate surgery and of preoperative chemotherapy discontinuation have not yet been established.

To alleviate these concerns, it is necessary to determine whether preoperative chemotherapy leads to substantially increased postoperative complications in patients who receive upfront chemotherapy followed by surgical resection. Several studies have recently reported on the practicality of upfront chemotherapy in unresectable metastatic colorectal cancer patients [2, 5, 15-17], finding an improvement of resectability after upfront chemother- apy in patients with metastatic colorectal cancer, with most procedures performed in patients with liver metastasis. None of the studies focused on short-term outcomes in patients with metastatic colorectal cancer who underwent surgery after upfront chemotherapy. Thus, the present study has several strengths. This study was performed only in patients with solid organ metastatic colorectal cancer and those with peritoneal metastasis. Chemotherapeutic agents used in treatment were modern protocols, reflecting the use of current standards of practice. Furthermore, this study specifically investigated complications known to be associated with chemotherapeutic agents.

In this investigation, we reviewed postoperative clinical course and complications in patients who underwent surgical resection after upfront chemotherapy. We compared the results obtained with those of patients who underwent surgical resection first and investigated whether there were any substantive differences between the 2 groups. In terms of postoperative short-term clinical outcomes, there was no difference between the 2 groups. Postoperative complications developed in $46.3 \%$ patients in the surgery first group and $60.6 \%$ patients in the upfront chemotherapy group. Most of the complications were minor, and there was no difference between the 2 groups in grade III or more major complications (surgery first group: $18.9 \%$ vs. upfront chemotherapy group: $27.5 \%$; $=0.374$ ).

It is well-known that oxaliplatin increases the risk of sinusoidal obstruction syndrome [18]. A recent publication from Volk et al. [19] demonstrated that preoperative administration of bevacizumab is associated with a strong reduction of total sinusoidal obstruction syndrome incidence. In that report, they also found that, while there was no difference in perioperative morbidity and mortality, there was a significant increase in wound complications in patients who received bevacizumab. In addition, a study by Vauthey et al. [20] revealed an increase in postoperative morbidity rates in patients who developed steatohepatitis. In their study, although there was no association between type of chemotherapy and postoperative complications, there was a trend toward increased postoperative morbidity in patients who underwent a longer duration of chemotherapy. Importantly, in this study, there was no significant increase in hepatobiliary or wound complications, bowel anastomotic leakage, or postoperative bleeding in patients in the upfront chemotherapy group. Although the upfront chemotherapy group had a higher incidence of postoperative ascites, these patients were more unresectable than those in the surgery first group due to the presence of more advanced liver metastatic lesions at the time of diagnosis. This led to more major liver resection after chemotherapy compared to patients in the surgery first group. Furthermore, in all cases, ascites was well-controlled with diuretic use alone. In our study, there was no increase in liver-related complications, especially in the upfront chemotherapy group. This occurrence may be due to the beneficial effects of preoperative bevacizumab administration mentioned by Volk et al. [19] or to development and implementation of postoperative 
care and surgical techniques. In this regard, further investigations will be needed. In our study, major liver resection (i.e., involving more than 4 segments) was performed in $21.5 \%$ of the upfront chemotherapy group. In 4 patients, the associating liver partition and portal vein ligation in staged hepatectomy approach were performed in relation to the extent of hepatic metastasis. There were no liver-related complications noted in our study.

There was no bleeding or anastomotic leakage in patients who received bevacizumab. This finding may support the idea that there is no need to delay surgery due to concern for toxicity caused by chemotherapy or the postoperative bleeding or anastomotic leakage known to be increased by bevacizumab.

In our study, upfront chemotherapy did not significantly increase postoperative complications. In particular, the incidence of specific complications known to be increased by chemotherapy was not substantially greater. These results were possible because of careful patient selection and appropriate treatment planning through a multidisciplinary team approach.

Our study has several limitations to consider. Since this study is a retrospective analysis of a single institution's experience with a small number of patients, we cannot make definitive conclusions about the safety and feasibility of upfront chemotherapy. In addition, it is difficult to assess the number and severity of complications correctly in a retrospective study. Furthermore, there is controversy regarding the definition of resectability. Although the treatment plan was chosen by a multidisciplinary team approach, without clear criteria, we determined resectability based on extent of metastatic disease and functional reserve of the remnant organ region. However, this study addresses the simple but important topic of evaluation of the association between upfront chemotherapy and postoperative morbidity in patients with metastatic colon cancer and may be helpful in decision-making for patients who require surgery after upfront chemotherapy.

In this study, we demonstrated that postoperative complications were not particularly increased in metastatic colon cancer patients who received upfront chemotherapy followed by curative surgery. There was no significant increase in hepatotoxicity, anastomotic leakage, or postoperative bleeding complications known to be associated with systemic chemotherapy. In these patients, focusing on the toxicity of chemotherapy should not interfere with the surgical decision in cases where surgery is necessary. Upfront chemotherapy followed by curative surgery in well-selected metastatic colon cancer patients is a possible treatment option in terms of safety and feasibility. Careful patient selection and treatment planning are important, including consideration of patient comorbidities and extent of surgery, through the multidisciplinary team approach.

\section{CONFLICT OF INTEREST}

No potential conflict of interest relevant to this article was reported.

\section{REFERENCES}

1. Edwards MS, Chadda SD, Zhao Z, Barber BL, Sykes DP. A systematic review of treatment guidelines for metastatic colorectal cancer. Colorectal Dis 2012;14:e31-47.

2. Adam R, Delvart V, Pascal G, Valeanu A, Castaing D, Azoulay D, et al. Rescue surgery for unresectable colorectal liver metastases downstaged by chemotherapy. In: Transactions of the Meeting of the American Surgical Association 2004;CXXII:242-56. https:// doi.org/10.1097/01.sla.0000141198.92114.f6.

3. Dehal A, Graff-Baker AN, Vuong B, Fischer T, Klempner SJ, Chang SC, et al. Neoadjuvant chemotherapy improves survival in patients with clinical T4b colon cancer. J Gastrointest Surg 2018; 22:242-9.

4. Niitsu H, Hinoi T, Shimomura M, Egi H, Hattori M, Ishizaki Y, et al. Up-front systemic chemotherapy is a feasible option compared to primary tumor resection followed by chemotherapy for colorectal cancer with unresectable synchronous metastases. World J Surg Oncol 2015;13:162.

5. Bogach J, Levine O, Parpia S, Valencia M, Ruo L, Serrano P. Does the addition of biologic agents to chemotherapy in patients with unresectable colorectal cancer metastases result in a higher proportion of patients undergoing resection? A systematic review and meta-analysis. J Gastrointest Surg 2018;22:523-8.

6. Garritano S, Selvaggi F, Spampinato MG. Simultaneous minimally invasive treatment of colorectal neoplasm with synchronous liver metastasis. Biomed Res Int 2016;2016:9328250.

7. Schmoll HJ, Van Cutsem E, Stein A, Valentini V, Glimelius B, Haustermans K, et al. ESMO Consensus Guidelines for management of patients with colon and rectal cancer. a personalized approach to clinical decision making. Ann Oncol 2012;23:2479-516.

8. Bilchik AJ, Poston G, Curley SA, Strasberg S, Saltz L, Adam R, et al. Neoadjuvant chemotherapy for metastatic colon cancer: a cautionary note. J Clin Oncol 2005;23:9073-8.

9. Kim CW, Baek JH, Choi GS, Yu CS, Kang SB, Park WC, et al. The role of primary tumor resection in colorectal cancer patients with asymptomatic, synchronous unresectable metastasis: study protocol for a randomized controlled trial. Trials 2016;17:34.

10. Shanbhogue AK, Karnad AB, Prasad SR. Tumor response evaluation in oncology: current update. J Comput Assist Tomogr 2010; 34:479-84.

11. Compton CC, Byrd DR, Garcia-Aguilar J, Kurtzman SH, Olawaiye A, Washington MK. Colon and rectum. In: Compton CC, Byrd DR, Garcia-Aguilar J, Kurtzman SH, Olawaiye A, Washington MK, editors. AJCC Cancer Staging Atlas: a companion to the Seventh Editions of the AJCC Cancer Staging Manual and Handbook. New York: Springer New York; 2012. p. 185-201.

12. Dindo D, Demartines N, Clavien PA. Classification of surgical complications: a new proposal with evaluation in a cohort of 6336 patients and results of a survey. Ann Surg 2004;240:205-13.

13. Lowe MC, D’Angelica MI. Anatomy of hepatic resectional surgery. Surg Clin North Am 2016;96:183-95. 


\section{Coloproctology Myung Hyun Han, et al.}

14. Khan H, Khan N, Ahmad A, Olszewski AJ, Somasundar P. Surgical management of metastatic colon cancer: A population-based analysis. J Geriatr Oncol 2015;6:446-53.

15. Chen HH, Lin JK, Chen JB, Chuang CH, Liu MC, Wang JY, et al. Neoadjuvant therapy of bevacizumab in combination with oxaliplatin and capecitabine (XELOX) for patients with metastatic colorectal cancer with unresectable liver metastases: a phase II, open-label, single-arm, noncomparative trial. Asia Pac J Clin Oncol 2018;14:61-8.

16. Devaud N, Kanji ZS, Dhani N, Grant RC, Shoushtari H, Serrano $\mathrm{PE}$, et al. Liver resection after chemotherapy and tumour downsizing in patients with initially unresectable colorectal cancer liver metastases. HPB (Oxford) 2014;16:475-80.

17. Folprecht G, Gruenberger T, Bechstein WO, Raab HR, Lordick F, Hartmann JT, et al. Tumour response and secondary resectability of colorectal liver metastases following neoadjuvant chemotherapy with cetuximab: the CELIM randomised phase 2 trial. Lancet Oncol 2010;11:38-47.

18. Rubbia-Brandt L, Audard V, Sartoretti P, Roth AD, Brezault C, Le Charpentier M, et al. Severe hepatic sinusoidal obstruction associated with oxaliplatin-based chemotherapy in patients with metastatic colorectal cancer. Ann Oncol 2004;15:460-6.

19. Volk AM, Fritzmann J, Reissfelder C, Weber GF, Weitz J, Rahbari NN. Impact of Bevacizumab on parenchymal damage and functional recovery of the liver in patients with colorectal liver metastases. BMC Cancer 2016;16:84.

20. Vauthey JN, Pawlik TM, Ribero D, Wu TT, Zorzi D, Hoff PM, et al. Chemotherapy regimen predicts steatohepatitis and an increase in 90-day mortality after surgery for hepatic colorectal metastases. J Clin Oncol 2006;24:2065-72. 